\title{
Phase diagram for the Harper model of the honeycomb lattice
}

\author{
Geo Jose, ${ }^{1}$ Rajesh Malla, ${ }^{1}$ Varadharajan Srinivasan, ${ }^{2,1}$ Auditya Sharma, ${ }^{1}$ and Suhas Gangadharaiah ${ }^{1}$ \\ ${ }^{1}$ Department of Physics, Indian Institute of Science Education and Research, Bhopal, India \\ ${ }^{2}$ Department of Chemistry, Indian Institute of Science Education and Research, Bhopal, India
}

(Dated: April 22, 2022)

\begin{abstract}
The Harper equation arising out of a tight-binding model of electrons on a honeycomb lattice subject to a uniform magnetic field perpendicular to the plane is studied. Contrasting and complementary approaches involving von Neumann entropy, fidelity, fidelity susceptibility, multifractal analysis are employed to characterize the phase diagram. The phase diagram consists of three phases: two metallic phases and an insulating phase. A variant model where next nearest neighbor hopping is included, exhibits a mobility edge and does not allow for a simple single phase diagram characterizing all the eigenstates.
\end{abstract}

PACS numbers: 71.10.Pm

\section{INTRODUCTION}

The localization of electronic states in a random medium due to quantum interference effects is known as Anderson localization [1. A consequence of this phenomenon is insulating behaviour in a one-dimensional (1D) lattice of non-interacting electrons subject to a random potential [2]. It is also the driving mechanism behind the metal-insulator transition (MIT) in disordered materials in 3D 24. However, for quasi-periodic systems such as the Aubry-Andre-Harper model (AAH) it is well known that a metal-insulator phase transition is exhibited even in 1D [5, 6].

The AAH model is obtained from a 1D tight-binding Hamiltonian and is given by

$$
t_{i+1} \phi_{i+1}+t_{i-1} \phi_{i-1}+V_{i} \phi_{i}=E \phi_{i}
$$

where $t_{i}, V_{i}$ and $\phi_{i}$ are the hopping terms, the on-site potential term and the wave function at the $i^{\text {th }}$ site, respectively. For the choice $t_{i}=1$ and $V_{i}=\lambda \cos (2 \pi \phi i+\theta)$ the above equation is derivable from the $2 \mathrm{D}$ square lattice nearest-neighbor hopping model in the presence of a uniform magnetic field 7]. For irrational values of $\phi$, the potential term $V_{i}$ is quasi-periodic and exhibits a localization-delocalization transition at $\lambda=2$ [6]. Below this value all the eigenstates are extended, while they are all localized for $\lambda>2$. The wave-functions at $\lambda=2$ are critical, exhibiting features such as multi-fractality.

Generalisations of the above model (extended Harper model) include next-nearest-neighbor (NNN) hopping on a square lattice and nearest-neighbour hopping on a twodimensional triangular lattice both in the presence of a uniform magnetic field [8]. Interestingly, in both these cases, the phase diagram consists of one localized phase and two metallic phases separated by critical lines and one bicritical point where the three phases meet. Over the years a number of techniques have been used to characterize the phases. Analytical techniques include calculation of the Lyapunov exponent [9] and measure of the spectrum [10]. Numerical techniques include the study of level statistics, in particular the distribution

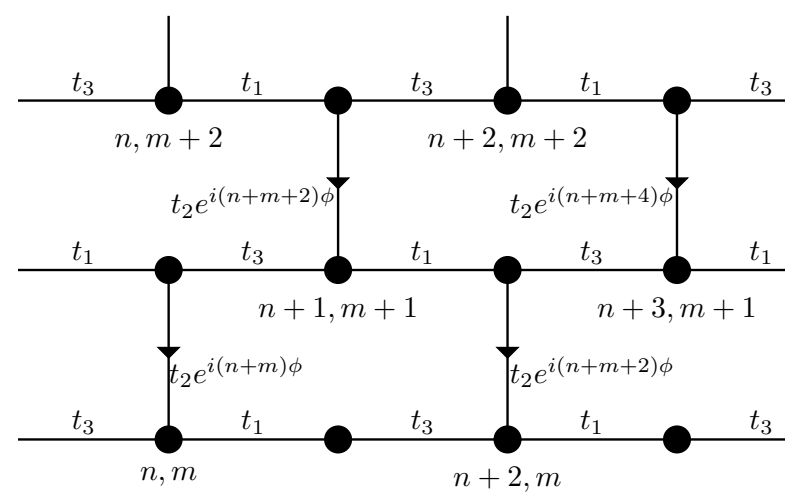

FIG. 1: Honeycomb lattice with nearest neighbor hopping in the presence of a uniform magnetic field.

of normalised energy gaps and bandwidths at the critical lines 11-13]; in addition multifractal analysis of the spectrum and the wave-function have proved useful in characterising the critical regions [14, 15].

In recent years the application of quantum information techniques in the context of quantum-phase transition (QPT) of a many-body system has led to unique insights [16, 17. In this regard the study of quantum fidelity and fidelity susceptibility have been quite useful in a system where the knowledge of the relevant order parameter and the changes in symmetries are not known 18. Quantum fidelity (QF) measures the overlap between two ground states with differing parameters, whereas fidelity susceptibility measures the change in QF between two parameters with infinitesimal separation [16 19]. Another important tool is the von Neumann entropy (VNE) which quantifies the entropy of entanglement [20, 21]. These techniques have been implemented to successfully characterise the phase diagram of the extended Harper model obtained by considering a triangular lattice 22.

On the experimental front, tremendous advancement, especially in the ability to use ultra cold atoms and photonic lattices for controlled simulation of many-body states, has created a lot of excitement [23, 24. Signatures 
of the localization-delocalization transition in the $\mathrm{AAH}$ model were observed almost a decade ago 25,26 . More complicated models are being simulated with the help of Raman lasers with inhomogeneous intensity 27, 28, lattice modulation techniques [29, 30] and laser assisted tunneling [31, 32. These techniques provide non-trivial Berry phases which serve as artificial gauge fields. The latter technique has been utilized to generate large tunable artificial magnetic fields and spatially dependent complex tunneling amplitudes which were successfully used to simulate the 2D square lattice Hofstadter model [33, 34]. With work being done on generating artificial honeycomb lattices for atoms via optical trapping techniques [35, 36, it can be expected that the 2D honeycomb Hofstadter model will be realized soon.

The energy spectrum of the honeycomb lattice with asymmetric hopping has been shown to exhibit the Hofstadter butterfly pattern [37. In this work, we obtain the phase diagram of the honeycomb lattice with asymmetric nearest neighbour hopping in the presence of a uniform magnetic field. With the help of quantum-information techniques, we show that similar to the case of the triangular lattice (and square lattice with NNN hopping), the phase diagram is divided into an insulating region and two metallic regions. However, in contrast to the other two cases, the phase diagram is different in structure. Further, multi-fractal analysis and level statistics approaches allow us to show that the lines separating the three regions are critical. In the last part of this work we include NNN hopping; this results in the appearance of mobility edges.

The organization of this paper is as follows. The next section describes the model, and reports the phase diagram obtained. The third section provides details of the quantum information techniques used to arrive at the phase diagram. The fourth and fifth sections are devoted to multi-fractal analysis and level statistics approaches respectively, for characterizing the critical lines. This is followed by a section on the NNN hopping model, and a final section of summary and conclusions.

\section{MODEL}

The tight binding Hamiltonian of an electron in a honeycomb lattice in the presence of a uniform magnetic field has the form:

$$
H=-\sum_{n+m=\text { even }}\left[t_{1} a_{n+1, m}^{\dagger} a_{n, m}+t_{2} \mathrm{e}^{-i(n+m) \phi} a_{n, m+1}^{\dagger} a_{n, m}++t_{3} a_{n-1, m}^{\dagger} a_{n, m}\right]+h . c .,
$$

where $a_{n, m}^{\dagger}\left(a_{n, m}\right)$ are the creation (annihilation) operators at the $(n, m)^{\text {th }}$ site, $t_{1}, t_{2}$ and $t_{3}$ are real hopping parameters, the gauge choice is $[0, \phi(x+y), 0]$, and $2 \phi$ is the flux enclosed per unit cell [Fig (1]]. For $\phi=p / q$, where $p$ and $q$ are coprimes, the phase term repeats itself after $q(2 q)$ sites along the $x$-direction for $q$ even (odd). The phase factor is attached with the $t_{2}$ hopping term and for fixed $n+m$ remains invariant for hopping from $(n, m) \rightarrow(n, m+1)$ site, consequently, the wave-function amplitude at the $(n, m)^{t h}$ site can be written as $\Psi_{n, m}=$ $\Psi_{n+m} e^{i\left(k_{x} n+k_{y} m\right)}$. Therefore the Harper matrix obtained from the Schrödinger equation, $\tilde{H}\left|\varepsilon, k_{x}, k_{y}\right\rangle=\epsilon\left|\varepsilon, k_{x}, k_{y}\right\rangle$, where $\left|\varepsilon, k_{x}, k_{y}\right\rangle=\sum \Psi_{n+m} e^{i\left(k_{x} n+k_{y} m\right)}|n, m\rangle$, acquires the following form 38

$$
\left(\begin{array}{cccccc}
-E & B_{1} & 0 & \cdots & 0 & B_{m}^{\prime *} \\
B_{1}^{*} & -E & B_{1}^{\prime} & \cdots & 0 & 0 \\
\vdots & \ddots & \ddots & \cdots & \vdots & \vdots \\
0 & 0 & \cdots & 0 & -E & B_{m} \\
B_{m}^{\prime} & 0 & \cdots & 0 & B_{m}^{*} & -E
\end{array}\right)=0
$$

where $m=q / 2$ for $q$ even, $m=q$ for $q$ odd, $B_{n}=$ $-t_{1} e^{i k_{x}}-t_{2} e^{i k_{y}} e^{i 4 \pi n \phi}$ and $B_{n}^{\prime}=-t_{3} e^{i k_{x}}$.

The corresponding 1D Harper Hamiltonian has the form of a non-interacting chain with asymmetric nearestneighbor hopping:

$$
\tilde{H}=\sum_{s} \Gamma_{s}^{l} c_{2 s-1}^{\dagger} c_{2 s}+\Gamma_{s}^{r} c_{2 s+1}^{\dagger} c_{2 s}+\text { h.c. }
$$

where the left and the right hopping parameters are $\Gamma_{s}^{l}=$ $-t_{1} \mathrm{e}^{i k_{x}}-t_{2} \mathrm{e}^{i k_{y}+i 4 \pi s \phi}$ and $\Gamma_{s}^{r}=-t_{3} \mathrm{e}^{-i k_{x}}$, respectively. Invoking the Schrödinger equation, $\tilde{H}|\psi\rangle=\epsilon|\psi\rangle$, where $|\psi\rangle=\sum \psi_{s} c_{s}^{\dagger}|0\rangle$, the following eigenfunction equations are obtained:

$$
\begin{aligned}
\epsilon \psi_{2 s} & =\Gamma_{s}^{l^{*}} \psi_{2 s-1}+\Gamma_{s}^{r *} \psi_{2 s+1} \\
\epsilon \psi_{2 s+1} & =\Gamma_{s+1}^{l} \psi_{2 s+2}+\Gamma_{s}^{r} \psi_{2 s}
\end{aligned}
$$

We re-express Eq. 5 in terms of the parameters $\mu=t_{1} / t_{3}$ and $\lambda=t_{2} / t_{3}$ and study the phase diagram as a function of $\mu$ vs $\lambda$ for irrational values of $\phi$. We fix $\phi$ to be the inverse of the golden ratio which we approximate as $\phi_{n}=F_{n-1} / F_{n}$, where $F_{n}$ is the $n$th number of the Fibonacci series. We find that the phase diagram, as shown in Fig (2), can be divided into three distinct regions. Each of the three phases is separated from the other by 


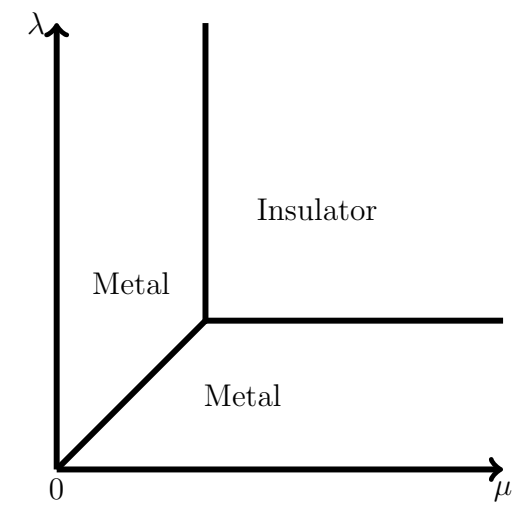

FIG. 2: The phase diagram as a function of $\mu$ vs $\lambda$. In the regions I and II the wavefunctions are all delocalized, whereas all states are localized in region III. The phases are separated by critical lines $\mu=\lambda, \mu=1$ and $\lambda=1$ which meet at $(1,1)$.

a critical line. These lines meet at the bicritical point, which corresponds to all the tunnelling parameters being identical. To characterise these regions we have used multiple techniques. While VNE, fidelity and fidelity susceptibility are able to characterise the regions into insulating or metallic type, we find that multi-fractal analysis and level statistics approaches are especially helpful for studying the wavefunction and the spectral properties at the critical lines.

We would like to add that unlike the various versions of the AAH model which have a quasiperiodic on-site potential term, our effective 1D model can be thought of as a modified version of the Su-Schrieffer-Heeger model with a modulating hopping term. In this work we have focussed on the ground state as well as bulk state properties of the wave-function to obtain the phase diagram and not on the edge states. The study of edge states by characterizing the gap via the calculation of the bulk Chern number or by counting the number of states between the band gap reveal the topological properties of the model. For a given set of parameters, the topological features undergo drastic modification with change in $\phi$ 37. However, the phase diagram we discuss are robust to changes in $\phi$ as long as it can be approximated as an irrational number.

\section{PHASE DIAGRAM}

\section{A. von Neumann Entropy}

We begin our analysis by studying the von Neumann entropy of the $\mathrm{N}$-site wave function averaged over all sites [22]:

$$
S_{j}=-\sum_{i=1}^{N} \frac{p_{j}^{i} \log _{2} p_{j}^{i}+\left(1-p_{j}^{i}\right) \log _{2}\left(1-p_{j}^{i}\right)}{N},
$$

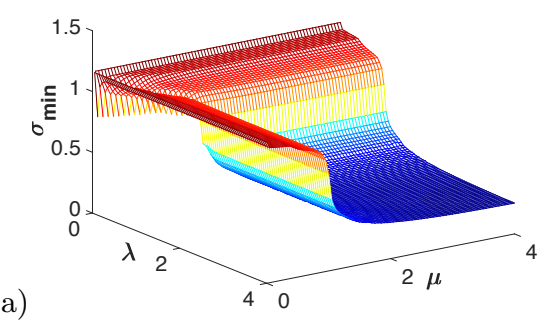

a)

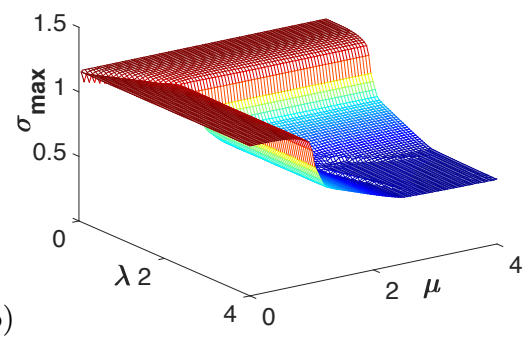

FIG. 3: Scaled entropy (Eq. 7) plots for $F_{n=12}$ as a function of the parameters $(\mu, \lambda)$ a) minimum and b) maximum entropy.

where $p_{j}^{i}$ is the occupation probability of the $i^{\text {th }}$ site of the $j^{\text {th }}$ wave-function. We note that for delocalised wave functions $p_{j}^{i} \sim 1 / N$ and hence $S_{j} \sim \log _{2}(N) / N$. On the other hand, $S_{j} \sim 1 / N$ for wave-functions that are localised. Thus the von Neumann entropy serves as an excellent diagnostic tool for distinguishing regions that are localised from those that are delocalised.

It is useful to define the scaled entropies as:

$$
\sigma_{j}=\frac{N}{\log _{2} N} S_{j}
$$

In Figs. $3(a)$ and $(b)$, we plot the minimum and maximum scaled entropy from the set $\left\{\sigma_{1}, \ldots, \sigma_{N}\right\}$ at each parameter value $(\mu, \lambda)$. Interestingly, even for relatively small system sizes (for example $F_{n=12}$ ), a clear separation between the extended regions II, III and the localized region I, is seen. Another feature that is illustrated from the near identical phase diagrams of Figs. $3(a)$ and $(b)$ is that there are no mobility edges. The wave functions in a given parameter regime are either all localised or all delocalised.

\section{B. Fidelity and Fidelity Susceptibility}

Although the VNE approach is able to distinguish regions into metallic and insulating type, the distinction between the two metallic regions remain inconclusive. We find that fidelity and FS techniques are able to further characterise the metallic regions.

Fidelity is defined as the overlap of the ground-state wave-functions for differing parameters and is given by

$$
F\left(\Upsilon_{1}, \Upsilon_{2}\right)=\left|\left\langle\psi_{0}\left(\Upsilon_{1}\right) \mid \psi_{0}\left(\Upsilon_{2}\right)\right\rangle\right|^{2},
$$

where $\left|\psi_{0}\left(\Upsilon_{i}\right)\right\rangle$ are the ground state wave-functions with parameters $\Upsilon_{i}=\left(\mu_{i}, \lambda_{i}\right), i \in\{1,2\}$. Keeping one of the 
a)

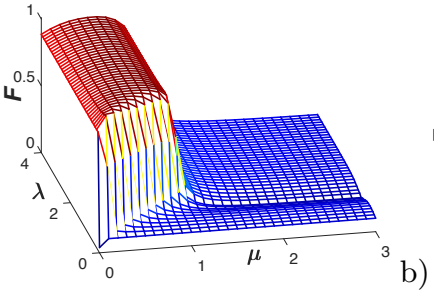

b)

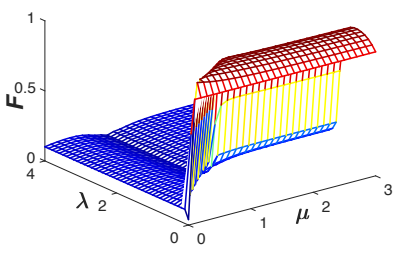

c)

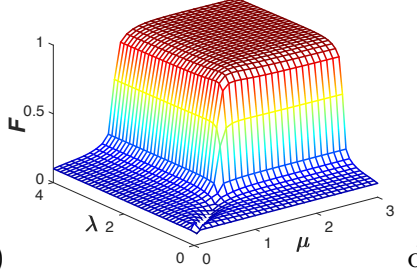

d)

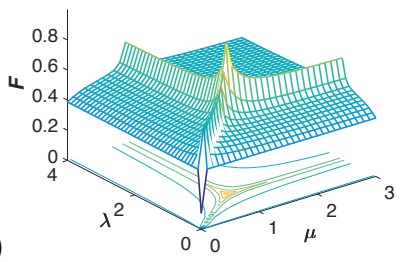

FIG. 4: Fidelity plots for $p / q=144 / 233$ with the reference paramters $(\mu, \lambda)$ : a) $(0.5,1)$, b) $(1,0.5)$, which are in the metallic regions 1 and 2 , respectively, c) $(2,2)$ in the insulating regime 3 and $d)$ the reference point $(1,1)$ which is the bicritical point.

parameters as the reference point, say $\Upsilon_{1}$, the other parameter $\Upsilon_{2}$, is varied. If the two parameters lie in the same phase the fidelity value is near unity. On the other hand, the ground-state wave-function differs significantly for parameters separated by a quantum critical point/line thus resulting in near vanishing fidelity values. In Figs. 4 (a), (b) and (c), we choose $(0.5,1),(1,0.5)$ and $(2,2)$ respectively, as the reference parameters. These highlight, respectively, insulating and the two metallic regions. Although the data presented is for the lowest energy state, we have checked that the same phase diagram is generated if arbitrary eigenstates are considered. The two metallic phases represent open orbits along different directions in the phase space of the underlying 'classicalHamiltonian' 13. An alternate insight into the various phases is obtained by studying limiting scenarios in Eqn. 4. For $\lambda=0$, the 1D Hamiltonian is metallic with unit-cell made of just two sites, whereas for the $\mu=0$ case, the unit cell is $\propto p$-sites and thus corresponds to an altogether different metallic phase. The $\mu \gg 1$ and $\lambda \gg 1$ regime is insulating since it is equivalent to cutting off of right-hopping from an even-site to an odd-site, thus generating localized islands.

Fidelity measurements can also highlight the critical lines. When the reference point is taken to be on a critical line, fidelity exhibits its maximum value along that line and a sharp decline away from it. This is best illustrated for the reference point $(1,1)$ (which is a bicritical point) for which fidelity exhibits peak behaviour [Figs. 4 d] along all the critical lines. The above approach to isolate the critical lines, is dependent on the correct positioning of the reference point. It turns out that an efficient alternate way is to use fidelity susceptibility where no guess work is required. Fidelity susceptibility is obtained from the fidelity $F(\Upsilon, \Upsilon+\delta \Upsilon)$ between the ground states of adjacent parameters [16, 19, 22]:

$$
\chi_{F}=\sum_{(a, b)=\lambda, \mu} \hat{n}_{a} \hat{n}_{b}\left[\frac{1}{2}\left(\left\langle\partial_{a} \psi_{0} \mid \partial_{b} \psi_{0}\right\rangle+\left\langle\partial_{b} \psi_{0} \mid \partial_{a} \psi_{0}\right\rangle\right)-\left\langle\partial_{a} \psi_{0} \mid \psi_{0}\right\rangle\left\langle\psi_{0} \mid \partial_{b} \psi_{0}\right\rangle\right]
$$

where the partial derivatives are in the parameter space and the unit vector $\hat{n}$ represents the direction connecting the two infinitesimally close points. The choice of the unit vector is arbitrary. If the unit vector is chosen to be parallel to one of the critical lines, then all critical lines except for the one parallel to the unit vector will be exposed. In our case, fidelity susceptibility plots obtained by considering atleast two choices of non-collinear unit vectors will reveal all the critical lines. In Fig. 5 we consider $(1 / \sqrt{3}, \sqrt{2 / 3})$ as the the unit vector; since this vector is not along any of the critical lines, only one plot is sufficient to highlight the position and directions of all the critical lines.

\section{Multifractal analysis}

In this section we will use multifractal techniques for analysing the scaling properties of wave functions in dif- ferent regions of parameter space. The basic idea is to express the probability measure of a wave-function at the $i^{\text {th }}$ site, $p_{i}$, in terms of a scaling index $\alpha_{i}$, such that $p_{i}=N^{-\alpha_{i}}$, where $N \propto F_{n}$ is the number of lattice sites at the $n^{t h}$ step. The distribution of $\alpha_{i}$ 's thus obtained can be organised in terms of the function $\Omega_{n}(\alpha)$ defined as the density of sites with scaling index $\alpha$, satisfying $\int \Omega_{n}(\alpha) d \alpha=N$. Further, $\Omega_{n}(\alpha)$ is expressed in terms of the scaling function $f_{n}(\alpha)$ such that $\Omega_{n}(\alpha)=N^{f_{n}(\alpha)}$.

With the increase in step-size $n$, the minimum of the scaling index $\alpha_{\min }$ approaches distinct limits for wavefunctions that are extended, localised and critical (for illustration see Fig. (6)). In particular, when the states are extended we expect that the probability measure at a typical point scale as $p_{i} \sim 1 / N$ and consequently $\alpha_{\min } \rightarrow 1$. On the other hand, the probability measure for localised states are concentrated at only few points where the measure is finite. Therefore for localised states $\alpha_{\min } \rightarrow 0$, whereas the points that are unoccupied result 


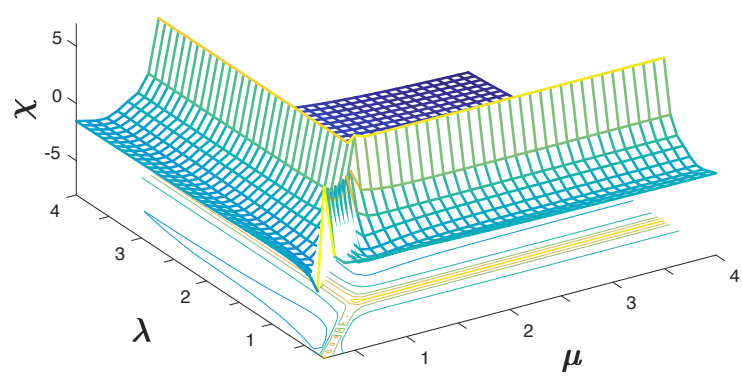

FIG. 5: Fidelity-susceptibility as a function of $(\mu, \lambda)$. The unit vector is taken to be along $\hat{n}=(1 / \sqrt{3}, \sqrt{2 / 3})$.

a)

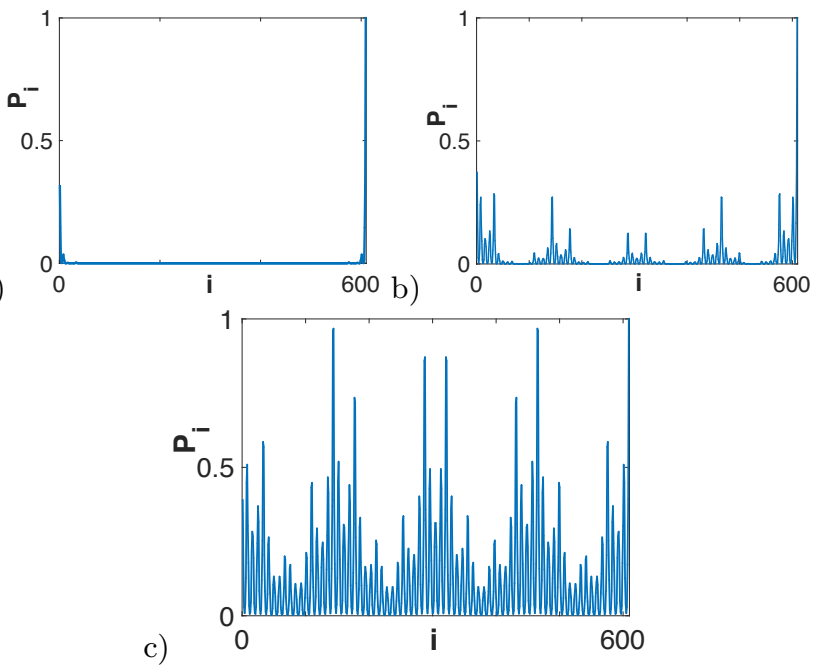

FIG. 6: Ground state wave-function $P_{i}=\frac{\left|\psi_{i}\right|^{2}}{\left|\psi_{\max }\right|^{2}}$ for $n=15$, a) in the insulating region $(1.1,1.1), b)$ at the bicritical point $(1,1)$ and, c) the metallic region $(0.9,1)$.

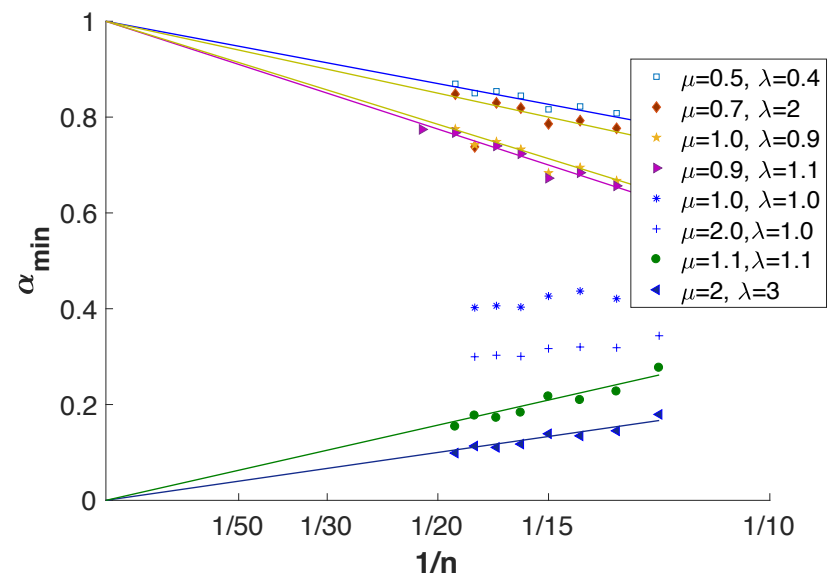

FIG. 7: $\alpha_{\min }$ vs $1 / n$. As $n \rightarrow \infty, \alpha_{\text {min }}$ acquires the limiting values 1 and 0 for metallic and insulating states, respectively. For critical states $\alpha_{\text {min }}$ approaches a value in between the two limits.
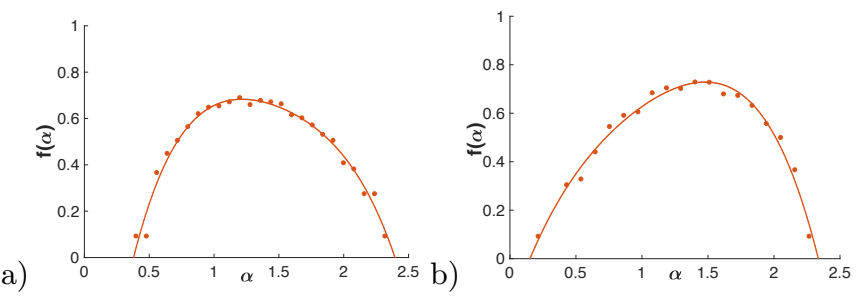

FIG. 8: $f(\alpha)$ vs $\alpha$ for $n=18$ at the critical points, a) $(1,1)$ and, b) $(2,1)$.

in $\alpha_{\max } \rightarrow \infty$. Similar arguments for the density of states suggest that for an extended state $f\left(\alpha_{\min }\right) \rightarrow 1$, while for a localised state $f\left(\alpha_{\min }\right) \rightarrow 0$ and $f\left(\alpha_{\max }\right) \rightarrow 1$. A critical state is characterised by $0<\alpha_{\min }<1$ and $f(\alpha)$ defined between the intervals $\left[\alpha_{\min }, \alpha_{\max }\right]$.

We consider the wave-functions at the lowest end of the spectra and numerically evaluate $\alpha_{\min }$ for Fibonacci indices $n$ and extrapolate them as $n \rightarrow \infty$. The plots of $\alpha_{\min }$ vs $1 / n$ are obtained for parameter points on the critical lines and away from it (see Fig. 7). The plots of $\alpha_{\min }$ vs $1 / n$ for the parameter points $(\mu, \lambda)=(0.5,0.4),(1,0.9)$ from metallic region I and $(\mu, \lambda)=(0.7,2),(0,9,1.1)$ from metallic region II, exhibit $\alpha_{\min } \rightarrow 1$ as $n \rightarrow \infty$ a behaviour consistent with extended states. For $(\mu, \lambda)=$ $(1.1,1.1),(2,3))$ which are in region III, $\alpha_{\min }$ approaches 0 as $n \rightarrow \infty$, which is what we expect for an insulating phase. Finally, we consider the behavior at the critical points $(\mu, \lambda)=(1,1)$ and $(2,1)$; we see from Fig. 7 that in the $n \rightarrow \infty$ limit, the exponent $\alpha_{\min }$ approaches a limit different from the metallic and insulating ones and lies between $0<\alpha_{\min }<1$. At the same time as seen in Fig. 8, $f(\alpha)$ acquires a dome like feature for the allowed values of $\alpha$ thus confirming the multifractal nature at the critical points [13].

\section{Level statistics}

As an alternative quantitative test for the universal features, we investigate the level statistics along the critical lines by studying the behavior of the spectral distribution function $P(\omega)$ as $\omega \rightarrow 0$. The normalizations used to define the distribution function are $\int_{0}^{\infty} P(\omega) d \omega=1$ and $\int_{0}^{\infty} \omega P(\omega) d \omega=\langle\omega\rangle=1$. We find that along the critical lines $P(\omega)$ diverges as $\omega^{-\delta}(\delta>0)$, a behaviour analogous to earlier studies on the gap distribution functions along critical lines for 1D quasi-periodic systems [13, 40]. 

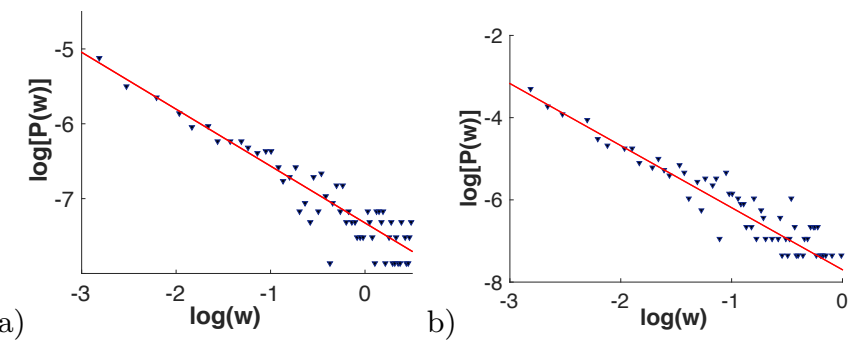

FIG. 9: Log-log plots for distributions of levels for a) $(\mu, \lambda)=$ $(0.5,0.5), \mathrm{b})(\mu, \lambda)=(1,2)$.

Fig. 9 shows the distribution of levels for $n=24$ and critical points $(\mu, \lambda)=(0.5,0.5)$ and $(\mu, \lambda)=(1,2)$. These can be fitted to the generic form:

$$
\log [P(w)]=-\delta \log (w)-\beta .
$$

For $(\mu, \lambda)=(0.5,0.5)$ and $(\mu, \lambda)=(1,2), \delta=1.52,1.51$ respectively. We obtain a similar plot for $(\mu, \lambda)=(1,1)$, with slope $\delta=1.52$. Thus along the critical lines the estimated value of $\delta$ is $\sim 1.5$, lending further support to universality along the critical lines.

\section{NEXT NEAREST NEIGHBOUR HOPPING}

In our study so far, we observe that all the eigenfunctions in each of the phases exhibit features similar to the corresponding ground state eigenfunction. In other words, the nearest neighbor hopping model we considered is characterised by a lack of mobility edge.

Here we will study the role of next-nearest-neighbor hopping on the localization-delocalization transition. For this purpose we will consider the following Harper equation [41]:

$$
E \Psi_{n}=D_{n} \Psi_{n}+R_{n} \Psi_{n+1}+R_{n-1}^{\dagger} \Psi_{n-1} .
$$

Here $D_{n}$ and $R_{n}$ are $2 \times 2$ matrices,

$$
D_{n}=\left(\begin{array}{cc}
a_{n} & c_{n} \\
c_{n}^{\dagger} & b_{n}
\end{array}\right), \quad R_{n}=\left(\begin{array}{cc}
d_{n} & 0 \\
f_{n} & e_{n}
\end{array}\right)
$$

where at $k_{x}=k_{y}=0$, the matrix entries are given in terms of the NNN hopping parameter $t$ and the NN hopping parameters $t_{1}, t_{2}, t_{3}$ as:

$$
\begin{aligned}
& a_{n}=2 t \sin [2 \pi(n-1 / 6) \phi], \quad b_{n}=-2 t \sin [2 \pi(n+1 / 6) \phi], \\
& d_{n}=-i t e^{i 2 \pi(n+1 / 3) \phi}\left(1-e^{i 2 \pi(n+1 / 3) \phi}\right), \\
& e_{n}=i t e^{i 2 \pi(n+2 / 3) \phi}\left(1-e^{i 2 \pi(n+2 / 3) \phi}\right), \\
& c_{n}=t_{2}+t_{1} e^{i 2 \pi \phi n}, \quad f_{n}=t_{3} e^{-i 2 \pi \phi(n+1 / 2)} .
\end{aligned}
$$

We note that our spinless Harper equation is a modified version of the one originally proposed by Beugeling et al. 41] for spinfull fermions with isotropic NN hopping and the NNN hopping terms being of the intrinsic spinorbit (ISO) and Rashba types, which correspond to spin conserving and non-conserving processes, respectively. In the intrinsic spin-orbit term, the up and down-spin terms have opposite sign in front of the tunnelling amplitude term. We have considered only the ISO type of hopping process corresponding to spinless fermions.

The effective one dimensional model as before has asymmetric NN hopping; in addition, the NNN hopping in the original hexagonal lattice manifests itself as an on site potential and NNN hopping along the chain. The Hamiltonian has the following form:

$H=\sum_{n}\left(a_{n} \alpha_{2 n}^{\dagger} \alpha_{2 n}+b_{n} \alpha_{2 n+1}^{\dagger} \alpha_{2 n+1}\right)+\sum_{n}\left(c_{n}^{*} \alpha_{2 n+1}^{\dagger} \alpha_{2 n}+f_{n-1} \alpha_{2 n-1}^{\dagger} \alpha_{2 n}+d_{n}^{*} \alpha_{2 n+2}^{\dagger} \alpha_{2 n}+e_{n}^{*} \alpha_{2 n+3}^{\dagger} \alpha_{2 n+1}+h . c.\right)(12$

In the absence of the NNN term we recover identically the earlier plots for the phase diagram, von Neumann entropy, fidelity and fidelity-susceptibility. However, turning on the NNN term leads to features that are not universal. In the absence of $t$, the fidelity plots for reference parameters $(\mu=2, \lambda=0.5)$ will reproduce Fig. 4 (b), however, when $t=0.08$, and 0.12 , the ground state fidelity yields plots as given in Fig. 10 (a) and (b), respectively. These plots are significantly different from each other as well as from the $t=0$ case, indicating strong dependence of the nature of the ground state on the NNN hopping parameter.
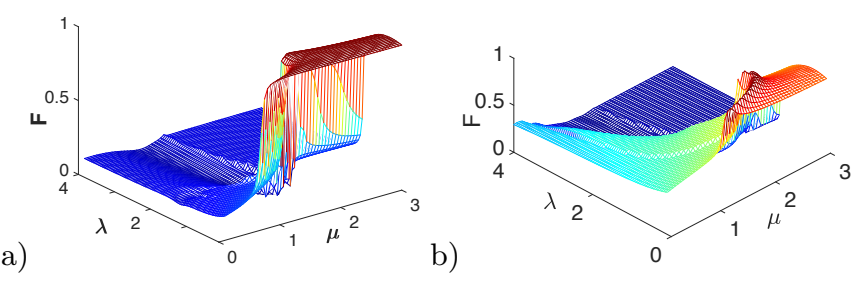

FIG. 10: Fidelity plots with $(\mu=2, \lambda=0.5)$ and next nearest hopping terms a) $t=0.08$ and b) $t=0.12$. 
a)
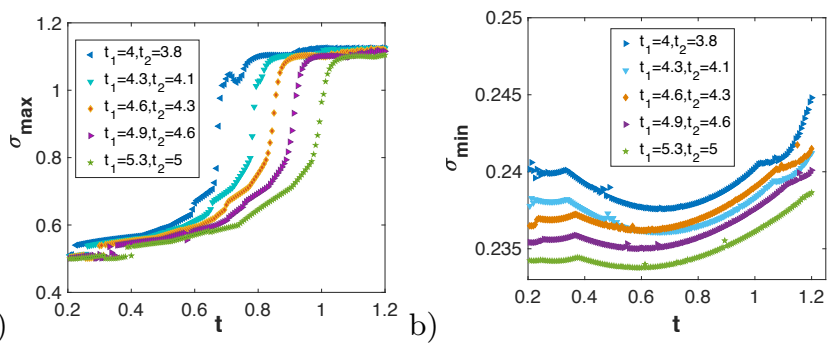

FIG. 11: Scaled entropy (Eq. 7) plots for the NNN model for a range of parameters $\left(t_{1}, t_{2}\right)$ as a function of the NNN coupling $t$. a) shows the maximum entropy among all the eigenstates. Suggests a transition from localized to delocalized nature. b) shows the minimum entropy, which do not indicate a transition in nature, within the given range.

It turns out that unlike the NN model, the ground state here is atypical; there are other eigenstates which are dramatically different from the ground state. To show this, we once again study the maximum and minimum values of the scaled entropy, just like in Fig. 3 for the NN problem, where $\sigma_{\min }$ and $\sigma_{\max }$ were qualitatively the same. In sharp contrast, Fig. 11 shows that in the presence of NNN coupling, the dependence as a function of $t$ of $\sigma_{\min }$ and $\sigma_{\max }$ are markedly different. As $t$ is increased there exists a critical $t_{c}$, dependent on the parameters $(\mu, \lambda)$, beyond which the states corresponding to the maximum entropy exhibit a localisation-delocalisation transition (for large $n$ the transition is sharper). For large $t, S_{\max } \sim \log _{2} N / N$ implying some of the states become delocalised. There is no such signature seen with $\sigma_{m i n}$. This is an indication of the presence of mobility edges in the spectrum.

A closer look at the plateau region of Fig. 11 a) is illuminating. Fixing $t$ to be in the plateau region (and $t_{1}=4, t_{2}=3.8$ ), let us study the scaled entropy for all the eigenstates as a function of energy. Two different cases of $t=1.2$, and $t=10$ are shown in Fig. 12. In each of these cases, it is possible to discern spread-out energy windows where $\sigma \sim 1$ corresponding to extended states, and other energy windows where $\sigma \ll 1$ corresponding to localized states. For $t=1.2$ a mobility edge separating localized and delocalized states can be identified to be around $\epsilon \approx 3$. However, such sequential behaviour is not universal as evidenced for $t=10$, wherein the behaviour exhibited is more complex. There are multiple closely separated energy bands at various energy scales wherein the wavefunctions manifest different behaviour: delocalized, localized and critical.

\section{CONCLUSIONS}

The phase diagram of the $2 \mathrm{D}$ honeycomb lattice with asymmetric nearest neighbour hopping in the presence of a uniform magnetic field is obtained, employing a variety of techniques. It is found to comprise of one insulating region and two metallic regions; von Neumann en-

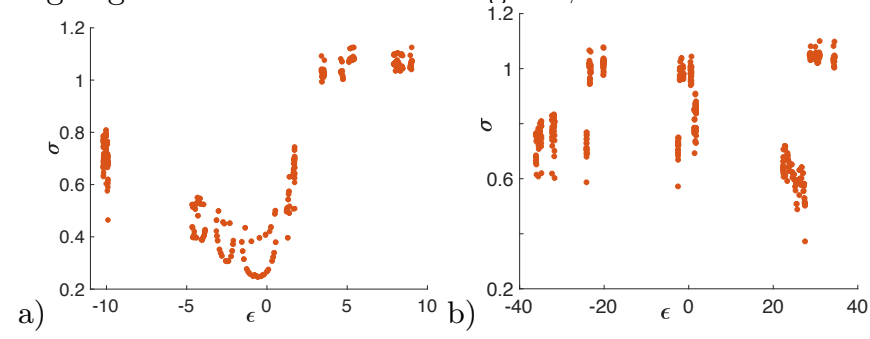

FIG. 12: Scaled entropy (Eq. 7) plots for all energies of the NNN model with a) $t=1.2$ and b) $t=10$.

tropy, averaged over all sites, is useful as a marker of the phase boundaries. The task of distinguishing between the two metallic phases, is accomplished with the help of fidelity, and fidelity susceptibility. An alternative perspective is provided by multifractal analysis, which allows for a finite-size scaling confirmation of the phase diagram. The study of level statistics reveals universal features along the critical lines. Finally, a variant Hamiltonian which incorporates next nearest neighbour hopping, is found to exhibit mobility edges in contrast to the earlier model.

While the present work has focussed on statics, a dynamical perspective is suggested by recent work 42 which has reported on certain driving protocols that can couple between groups of localized states and convert them into extended states. It would be interesting to explore such effects in our model on the honeycomb lattice, which provides a rich phase diagram. The possibility of many-body localization when interaction is turned on 43, and other many-particle effects [4], provides impetus for including interaction in our model. The AAH model has been shown to be topologically nontrivial [45]; it remains to be seen whether our $1 \mathrm{D}$ chain admits a topological phase.

\section{Acknowledgments}

S.G. would like to thank Diptiman Sen for helpful discussions. A.S and S.G. are grateful to SERB for the support via grants YSS/2015/001696 and EMR/2016/00264, respectively. We thank the HPC facility of IISER, Bhopal, where part of the computational work was carried out. 
[1] P. W. Anderson, Phys. Rev. 109, 1492 (1958).

[2] 50 Years of Anderson Localization, edited by E. Abrahams (World Scientific, Singapore, 2010).

[3] E. Abrahams, P. W. Anderson, D. C. Licciardello, and T. V. Ramakrishnan, Phys. Rev. Lett. 42, 673 (1979).

[4] M. Imada, A. Fujimori, and Y. Tokura, Rev. Mod. Phys. 70, 1039 (1998).

[5] P. G. Harper, Proc. Phys. Soc. Lond. A 68, 874 (1955).

[6] S. Aubry and C. Andre, Proc. Israel Phys. Soc. 3, 133 (1980).

[7] D. R. Hofstadter, Phys. Rev. B 14, 2239 (1976).

[8] J. H. Han, D. J. Thouless, H. Hiramoto, and M. Kohmoto, Phys. Rev. B 50, 11365 (1994).

[9] T. C. Halsey, M. H. Jensen, L. P. Kadanoff, I. Procaccia, and B. I. Shraiman, Phys. Rev. A 33, 1141 (1986).

[10] D. J. Thouless, Phys. Rev. B 28, 4272 (1983).

[11] S. N. Evangelou and J. L. Pichard, Phys. Rev. Lett. 84, 1643 (2000).

[12] Y. Takada, K. Ino and M. Yamanaka, Phys. Rev. E 70, 0662032004 .

[13] K. Ino and M. Kohmoto, Phys. Rev. B 73, 205111 (2006).

[14] M. Kohmoto, Phys. Rev. A 37, 1345 (1988).

[15] K. Ikezawa and M. Kohmoto, J Phys. Soc. of Japan, 63, 6, (1994).

[16] P. Zanardi and N. Paunkovic, Phys. Rev. E 74, 031123 (2006).

[17] H. T. Quan, Z. Song, X. F. Liu, P. Zanardi, and C. P. Sun, Phys. Rev. Lett. 96, 140604 (2006).

[18] Shi-Jian Gu, Intl. J. Mod. Phys. B, 24, 4371 (2010).

[19] W. L. You, Y. W. Li, and S. J. Gu, Phys. Rev. E 76, 022101 (2007).

[20] C. H. Bennett, H. J. Bernstein, S. Popescu, and B. Schumacher, Phys. Rev. A 53, 2046 (1996).

[21] A. Steane, Rep. Prog. Phys. 61, 117 1998; D. P. DiVincenzo and C. H. Bennett, Nature London 404, 2472000.

[22] L. Gong and P. Tong, Phys. Rev. B 78, 115114 (2008).

[23] M. Lewenstein, A. Sanpera, V. Ahufinger, B. Damski, A. Sen(De), and U. Sen, Adv. Phys. 56, 243 (2007).

[24] I. Bloch, J. Dalibard, and W. Zwerger, Rev. Mod. Phys. 80, 885 (2008)

[25] J. Billy et al., Nature (London) 453, 891 (2008).

[26] G. Roati et al., Nature (London) 453, 895 (2008).
[27] Y. Lin, R. L. Compton, K. Jimenez-Garca, J. V. Porto, and I. B. Spielman, Nature (London) 462, 628 (2009).

[28] J. Dalibard, F. Gerbier, G. Juzeliu nas, and P. O hberg, Rev. Mod. Phys. 83, 1523 (2011).

[29] J. Struck, C. Olschlager, M. Weinberg, P. Hauke, J. Simonet, A. Eckardt, M. Lewenstein, K. Sengstock, and P. Windpassinger, Phys. Rev. Lett. 108, 225304 (2012).

[30] J. Struck, M. Weinberg, C. O lschlager , P. Windpassinger, J. Simonet, K. Sengstock, R. Hoppner, P. Hauke, A. Eckardt, M. Lewenstein, and L. Mathey, Nat. Phys., doi: 10.1038/nphys2750 (2013).

[31] A. R. Kolovsky, Europhys. Lett. 93, 20003 (2011).

[32] C. E. Creffield and F. Sols, Europhys. Lett. 101, 40001 (2013).

[33] M. Aidelsburger, M. Atala, M. Lohse, J. T. Barreiro, B. Paredes, and I. Bloch, Phys. Rev. Lett. 111, 185301 (2013).

[34] H. Miyake, G. A. Siviloglou, C. J. Kennedy, W. C. Burton, and W. Ketterle, Phys. Rev. Lett. 111, 185302 (2013).

[35] M. Polini, F. Guinea, M. Lewenstein, H. C. Manoharan, and V. Pellegrini, Nature Nanotechnology 8, 625 (2013).

[36] S. Safaei, C. Miniatura, and B. Gremaud, PRA 92, 043810 (2015).

[37] G. Gumbs and P. Fekete Phys. Rev. B 56, 3787 (1997).

[38] Y. Hasegawa, Y. Hatsugai, M. Kohmoto, G. Montambaux, Phys. Rev. B 41, 1990.

[39] S. J. Gu, S. S. Deng, Y. Q. Li, and H. Q. Lin, Phys. Rev. Lett. 93, 086402 (2004).

[40] M. Naka, K. Ino, and M. Kohmoto, Phys. Rev. B 71, 245120 (2005).

[41] W. Beugeling, N. Goldman, and C. M. Smith, Phys. Rev. B 86, 075118 (2012).

[42] L. Morales-Molina, E. Doerner, C. Danieli, and S. Flach, Phys. Rev. A 90, 043630 (2014).

[43] R. Modak and S. Mukerjee, Phys. Rev. Lett. 115, 230401 (2015).

[44] L. Wang, N. Liu, S. Chen, and Y. Zhang, Phys. Rev. A 95, 013619 (2017).

[45] Y. E. Kraus and O. Zilberberg, Phys. Rev. Lett. 109, 116404 (2012). 